\title{
IDENTIFYING PREDICTORS OF DIFFICULT INTUBATION AMONG OBESE ORAL SURGICAL PATIENTS
}

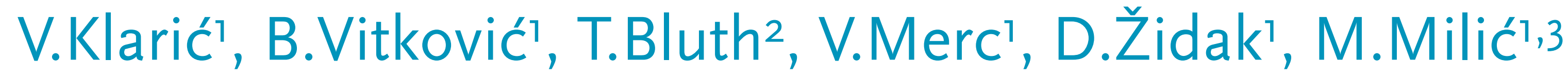 \\ 'Dpt of Anesthesiology and Intensive Care Medicine, University Hospital Dubrava, Zagreb, Croatia; ${ }^{2}$ Dpt of Anesthesiology and \\ Intensive Care Medicine, University Hospital Carl Gustav Carus, Dresden, Germany; ${ }^{3}$ University Dubrovnik, Croatia
}

1. INTRODUCTION:

- Obesity, defined as body mass index (BMI) between 30 and $39.9 \mathrm{~kg} / \mathrm{m}^{2}$, and extreme obesity, with $\mathrm{BMI}$ more than $40 \mathrm{~kg} / \mathrm{m}^{2}$ is a multisystem, chronic disorder associated with a significant increase in perioperative complications'.

- Obese patients undergoing oral surgery may provide a unique set of anesthestic challenges associated with airway management ${ }^{2}$.

- The aim of the study was to evaluate possible predictors of difficult intubation among obese patients scheduled for oral surgical procedures under general anesthesia.

2. PATIENTS AND METHODS:

- This was an explorative single-center prospective observational study.

- Following ethical approval and signed written informed consent, 75 obese patients (30-65 year old and ASA II-III) undergoing oral surgery were enrolled.

- The number of front teeth (incisors and canines), neck circumference (NC), waist-to-hip (W/H) ratio, BMI and the risk for postoperative pulmonary complications(PPC) determineted by ARISCAT score was assessed 3 .

- Difficult intubation was defined as it was possible after $>2$ attempts and/or using alternative techniques: Mc Coy laryngoscope, Gum elastic Bougi, fiber bronchoscope or Bonfils and assessed by two indpended investigators.

- Receiver operating curve (ROC) analyses were performed to identify predictors of difficult intubation and their cut-off values.

\section{RESULTS AND DISCUSSION:}

- The average BMI was 34.6 (SD 3.6), with 42 males. Average waist to hip $(\mathrm{W} / \mathrm{H})$ ratio was 1.1 (women 0.99 $+/-0.19$, males $1.11+/-0.18)$. The mean value of the NC was 54.2 (SD 6.9) cm, significantly higher in males $(p=0.003)$. Patients did not have increased risk of PPC (mean Ariscat score 9, range 0-31).

- Difficult intubation was observed in $15(20 \%)$ patients $(9.3 \%$ women, $10.7 \%$ males, $p=0,72)-$ conventional intubation possible in all patients.

- ROC analysis showed that values of NC above $57 \mathrm{~cm}$ had $86.7 \%$ sensitivity and $81.7 \%$ specificity in prediction of difficult intubation (area under the curve [AUC] $=0.918$ (Figure 1a, 1b), whereas other parameters showed no significant prediction.

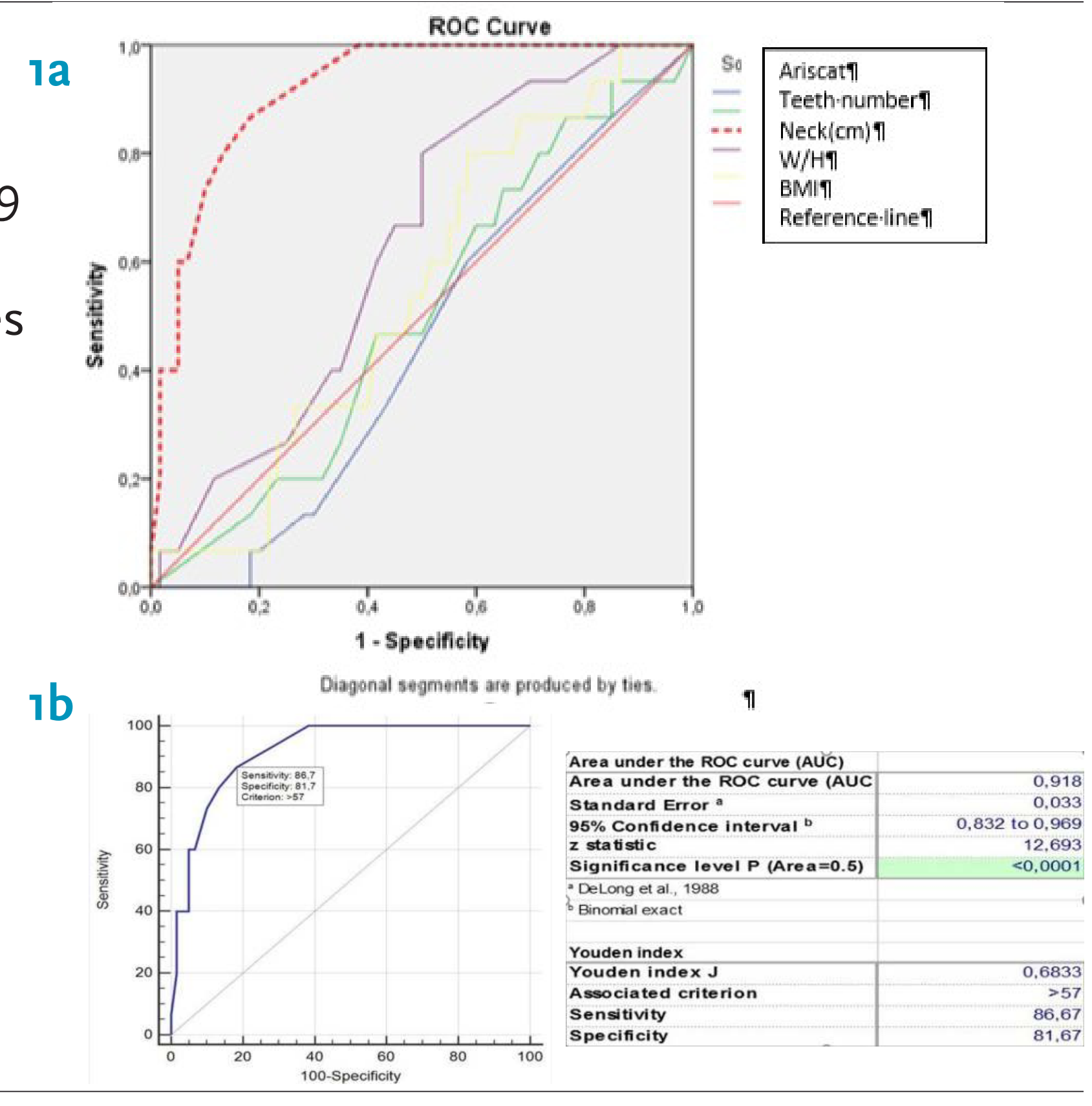

\section{CONCLUSION:}

- In our population of obese patients undergoing oral surgery we found that a neck circumference above $57 \mathrm{~cm}$ had high sensitivity and specificity in the prediction of difficult intubation.

- We recommend routine measurement of neck circumference in obese patients during preoperative assessment. 